\title{
Les innovations financières facteurs d'instabilité au cœur des crises financières Référence au cas algérien
}

\begin{abstract}
:
Financial innovation has long-term benefits because it facilitates the process of financial development. But there is a dark side in the development of complex financial products that are increasingly becoming more opaque and speculative, a point that was more motivated by the search of higher yields than by the desire to provide investors with hedging instruments. It is according to its effects and consequences that the subject of financial innovation is a present-day subject and worthy of attention. From the debate over the study and after setting a conceptual framework for studying the key role it played in the subprime crisis of 2008 and the crisis of European debt, we will examine the various consequences of these innovations on the Algerian economy.
\end{abstract}

Key words: financial innovation, securitization, subprime crisis, sovereign debt crisis, Algerian economy.

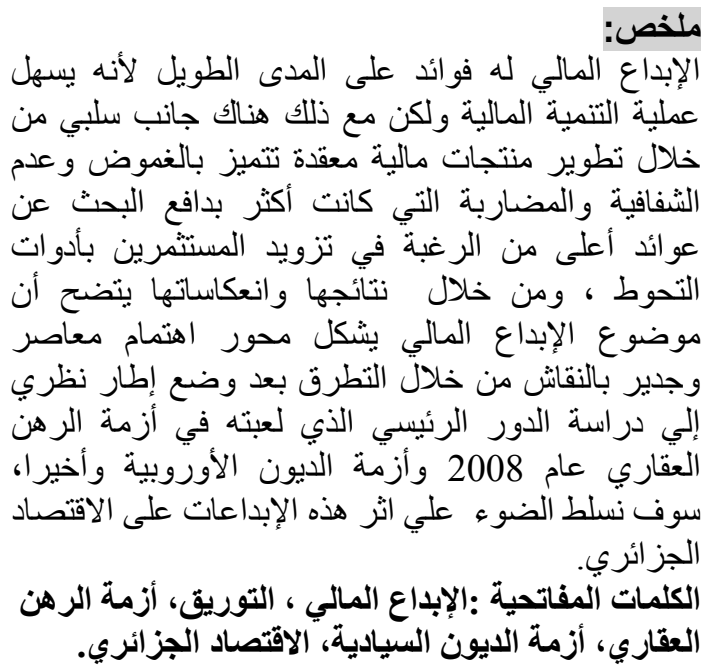

\section{ZEGHBID NASSIM LOTFI}

Pr. BENTABET Ali

Faculté des sciences économiques et sciences de gestion Université de Badji MOKHTAR Annaba 
De facto,les années précédant la crise ont été caractérisées par la création et le développement de produits financiersstructurés, technique qui consistait à élaborer des instruments financiers en utilisant différents sous-jacents dont les prêts subprimes. La crise a tout d'abord touché les produits structurés qui contenaient une partie de ces prêts subprimes, puis s'est propagée par effets de contagionà l'ensemble des produits structurés en raison des doutes liés à leur véritable composition.Si le recours à la titrisation a entraîné la prolifération de produits structurés très complexes ( $\mathrm{CDO}$ d'ABS, $\mathrm{CDO}$ de $\mathrm{CDO}$ ), il a également contribué à l'émergence d'un nouveauBusiness model pour les banques, qui ont abandonné leur rôle traditionnel d'octroi et de détention du crédit (intermédiaire financier) pour une nouvelle fonction d'octroi et de cession du crédit (trader) L'innovation financière et notamment les dérivés de crédit ont contribué à ce phénomène, en soutenant la croissance excessive du crédit et l'accumulation de risques de crédit, avec pour preuve l'accumulationpar l'ensemble du système financier d'importantes pertes. En outre, le manque de transparence des marchés (illisibilités-nonvisibilité)a certainement contribué à renforcercette prise de risques excessifs et a accentué les effets de contagion entre les intervenants de marché.

AIG et LehmanBrothersillustrent les risques systémiques inhérentsà une utilisation sans limite des produits dérivés.

Comme réplique, les États et les banques centrales ont aidé les systèmes bancaires à hauteur de plus de 14000 milliards de dollars, selon les estimations pour éviter les faillites et la crise systémique.Mais cette injection massive n'a pas pu empêcher une crise du crédit,provoquant une crise économique. Enfin, la crise économique s'est transformée à son tour en crise des dettes souveraines avec une chute importante de la croissance dans la plupart des pays développés. L'économie algérienne vu le faible taux d'intégration financière aux marchés financiers internationaux a relativement protégé son économie des effets directs de la crise financière. Elle s'est ainsi retrouvée à l'abri de l'impact direct de la crise des subprimes de 2008, ce qui lui a permis d'éviter les effets néfastes et destructeurs d'une crise systémique et financière qui a ébranlé les marchés financiers internationaux.Sauf que l'économie reste vulnérable compte tenu de l'instabilité récurrentedes marchés du pétrolé et du gaz, des biens alimentaires dont elle est très dépendante : irrémédiablement, le rythme de croissance de l'économie algérienne risque de se ralentir a défaut d'une ruelle capacité de sa résilienceface a des retournement brusques et durables des prix sur les dits marches.

Dans ce contexte, une question fondamentale se pose : comment L'innovation financière qui est sensé construire un système financier internationalsolide peut être une source de sa déstabilisation ? Et comment ces innovations ont pu affecter l'économie algérienne? 
Pour répondre à cette question, l'écrit se base sur une lecture théorique des concepts pour avancer une réflexion sur l'innovation financière et son interaction avec les crises financière.

La question cruciale est de montrer les interactions entre l'innovation financière dans le déclenchement des crises, Ensuite on essaye d'illustrer cette interaction par la décortication desproduits structurés et leurs rôle dans la crise financière de 2008, et des dettes européennes ainsi que les reformes qu'elle induit pour le système financier mondial.

Le dernier point de la réflexion porte sur l'étude de l'effet des innovations financièressur l'économie algérienne. Et de l'état actuelde l'économie nationale, dans un contexte d'instabilité de l'économie internationale.

I. Le cadre conceptuel de L'innovation financière :

1- définition de $L$ 'innovation financière :

L'innovation financière peut être définie comme l'ensemble de la procédure informationnelle, structurelle et technique par laquelle la banque vise à réduire les charges par L'automatisation des démarches bancaires, à abaisser le coût de collecte de l'information et de l'épargne auprès des agents, aussi à diminuer le risque lié à l'allocation des ressources(1).

L'innovation financière amène les banques à non seulement améliorer les produits financiers existants mais aussi à créer de nouveaux produits de manière à répondre de façon optimale à la demande des agents, aux besoins du marché, aux contrainteslégales et institutionnelles, et enfin, aux changements de l'environnementmacroéconomique Ainsi, puisque L'innovation financière joue un rôle primordial dans la promotion du capital risque, dans L'accroissement de la liquidité et dans le transfertdes risques, elle serait un maillon essentiel dans le processus de croissanceéconomique(2).

2- $\quad$ Les causes de $L$ 'innovation financière :

Nous identifions trois principales causes de L'innovation financière à savoir :

-Les contraintes réglementaires :

Silber (1983) explique que la principale raison de l'innovation financière est le contournement de la réglementation bancaire pour ce dernier l'innovation financière vise à réduire les contraintes financières qui pèsent sur les firmes,de nouvelles sources de mobilisation et d'utilisation des fonds sont inventées,puisque les changements exogènes de l'environnement stimulent la recherche de nouvelles politiques d'action dans la mesure où le développement de nouveaux produits financiersest coûteux, le stimulus de l'innovationfinancière peut être interprété comme un accroissement des coûts liés au respect des contraintes existante(3).

En effet, pour Abraham (1988), l'innovation financière est indispensable à la croissance puisqu'elle a été utilisée pour contenir l'explosion des coûts Dans le secteurfinancier et ensuitepour offrir denouvelles possibilités de profits dans unenvironnement, caractérisé paruneréglementation contraignante une 
demande plusdiversifiée de la partde la clientèle.Enfin une diminution des margesd'intermédiation compte tenu de l'exacerbation,dela concurrence bancairel'innovation financière selon Bhatt (1988) c'est également l'adaptation créative de la technologie bancaire à l'environnement économique(4).

-L'accroissement des coûts et la concurrence dans le secteur financier :

L'innovation financière naît également de la pression économique de la concurrence qui conduit à une amélioration, des fonctions du système financier selon Bodie et Merton (2001), et est le fruit des activités des entrepreneurs De ce fait, les forces économiques qui déterminent les innovations financières sont les mêmes que pour les innovations en général(5).

-Les problèmes d'asymétrie d'information :

Ross (1989) montre que l'innovation financière permet de réduire les problèmes d'asymétrie d'information par l'élaboration des produits financiers qui facilitent l'accès à l'information, ce qui réduit les coûts d'agence et de monitoring et estime aussi que l'innovation financière joue un rôle important dans l'économie en réduisant les coûts de transaction, en offrant un meilleur partage du risque et en réalisant des plus-values financières.

D'autres causes secondaires de L'innovation financière ont été identifiées l'innovation technologique accroît les parts de marché et entraîne L'innovation financière, les conditions macroéconomiques fluctuations des prix, des taux d'intérêt, des taux de change en effet, l'instabilité macroéconomique accroît L'innovation financière, la régulation financière (contraintes légales) ,la politique de taxation (trop de taxes impliquent L'innovation financière, les changements dans les conditions de l'environnement macroéconomique par exemple : la fin du régime de BrettonWoodset du change fixe au début des années 70(6).

II. Innovations et crises financière :

1- Le rôle de l'innovation financière dans la crise des subprimes:

On appelle subprimes des crédits hypothécaires accordés à des ménages à faible solvabilité aux États-Unis, l'encours des subprimes s'élevait à 1300 Mds \$ pour 6 millions de ménages, contre 330 Mds $\$$ quatre ans plus tôt.

Les prêts ont été accordés pour des durées comprises entre 25 et 30 ans, à des taux d'intérêt très bas les premières années (environ 1,5\%), puis indexés sur les taux du marché à partir de 2004, face à la réapparition des tension sinflationnistes, la Fed remonte régulièrement ses taux directeurs, les faisant passer de $1 \%$ à 5,25\%.fin 2006, les prix de l'immobilier chutent Les deux phénomènes, hausse des taux et chute des prixdansl'immobilier,concourent à alourdirconsidérablement les charges financières des ménages les plus démunis et ne leur permettent plus de couvrir l'intégralité de leur créance par la vente du bien concerné Les tauxdenon-remboursement de ces créditsatteint $15 \%$ en 2007 la baisse des prix de l'immobilier. 
Amène la valeur des biens en deçà de la valeur des crédits, la garantie apportée ne couvre plus la perte des banques qui font face à des déséquilibres importants dans leur bilan c'est le démarrage d'une crise de contrepartie classique qui aurait pu rester circonscrite aux banques hypothécaires américaines. Mais les créances ont été sorties du bilan de ces banques pour être rachetées par d'autres établissements financiers ou des fonds d'investissements aussi la simple crise de contre partie s'est-elle propagée de par les institutions financières et de par le monde à cause des opérations de titrisation(7).

-La titrisation ou le principe du transfert du risque :

La titrisation, apparue dans les années 70 , consiste à transformer des actifs non négociables sur les marchés, des crédits bancaires notamment en instruments de placement négociables adossés à ces actifs et permettant à l'émetteur d'obtenir des liquidités. La banque qui émet ce type d'obligations peut, au lieu de conserver les créances d'origine à son bilan, les céder à des investisseurs sur le marché avec les risques de défaut qui y sont associés Le mécanisme en soi est bénéfique car il permet à la banque de transférer certains risques sur des tiers et donc d'en retirer des ressources qu'elle pourra à nouveau prêter(8).

Le plus souvent, la banque à l'origine des prêts les cède à une banque d'investissement (SpecialPurposeVehicule, SPV) qui forme un pool de crédit structurés (Assetbacked Securities, ABS), homogènes ou hétérogènes (MBS Mortgage-BackedSecurities, CDO CollateralizedDebt Obligations) Les titres sont émisen tranches hiérarchisées selon leur niveau de risque. A partir d'un pool de MBS noté BBB par exemple, le véhicule parvient à proposer aux investisseurs des tranches de titres présentant des niveaux de risque et de rendement différentes : tranches « super senior » notées AAA, « senior » notées $\mathrm{AA}$ et $\mathrm{A}$, « mezzanine » notées $\mathrm{BBB}$ et $\mathrm{BB}$, jusqu'aux tranches " equity » non notéesLa tranche dite "equity" vient absorber l'essentiel du risque attaché au portefeuille d'actifs titrisés.

Les organismes de crédit hypothécaire et les banques allaient en effet faire franchir une nouvelle étape à la titrisation des crédits hypothécaires et de toute une série de créances assimilées désormais, il s'agissait pour les prêteurs originels de transférer le risque de non-remboursement sur le plus grand nombre dans le cadre d'un business model le modèle originate and distribute " initier et transmettre », lequel avait progressivement supplanté celui de la conservation du risque par son initiateur (originate and hold)(9).

Sachant que le risque pouvait être transféré grâce à la titrisation, les banques ont prêté une moindre attention à la solvabilité de leurs clients. La titrisation a entraîné une plus faible transparence des risques car les crédits subprimes ont été achetés et vendus àdes investisseurs nationaux et internationaux sans qu'ils connaissent réellement la qualité des titres possédés. La diminution de la valeur des titres a été provoquée par la crise immobilière qui a entraîné une crise de 
confiance.Le lien entre les $\mathrm{CDO}$, les ABS, et leurs sous-jacents est devenu tellement complexe qu'il est extraordinairement difficile de simuler l'impact sur une tranche de CDO d'un scénario affectant les sous-jacents (combien de propriétaires de maisons vont-ils faire défaut, et quelle sera la perte sur la valeur originelle du crédit qu'il faudra constater quand tout aura été réglé? comment ceci impactera-t-il les différentes tranches de risque que comprend le CDO ?)(10).

- Les dérives des agences de notation dans la crise des subprimes :

Les agences de notation sont l'un des boucs émissaires de la crise financière, Trois agences de notation, représentant plus de $80 \%$ du marché mondial du rating, Standard \& Poor's , Moody's Investor Service et Fitch Rating, émettent régulièrement des opinions sous forme de notation sur la capacité d'un emprunteur à faire face, à bonne date, aux échéances de remboursement en intérêts et capital de la dette contractée. Qui s'exprime selon une échelle de notation qui comprend une gamme de 21 à 23 notes, de la plus élevée (AAA pour Standard \& Poor's et Fitch Rating et Aaa pour Moody's) à la plus basse (D pour les deux premières et $\mathrm{C}$ pour la dernière)(11).

Les agences de notation furent accusées d'avoir encouragé la bulle spéculative sur les produits structurés en ne gardant pas une neutralité suffisante La principale critique est la relation particulière qui existe entre les agences de notation et les émetteurs (clients) qui débouche parfois sur des conflits d'intérêt. Si l'agence est rémunérée par l'entreprise émettrice, ce qui est plus souvent le cas, nous pouvons douter de l'indépendance de son jugement. En effet, les agences sont payées par ceux qu'elles notent. Ces dernières années les notations dans ces opérations (appelées notations de produits structurés) ont représenté jusqu'à $50 \%$ de leur chiffre d'affaires. En outre, le nombre de clients étant assez limité, les agences se sont sans doute retrouvées en situation de dépendance par rapport à leurs clients. De plus, pour ces produits, l'agence n'intervient pas seulement comme évaluateur d'une entreprise qui existe déjà mais comme conseillère d'une opération en cours de montage. Elle fait partie du processus qui constitue le produit. Elle mélange activité de conseil et notation(12).

-Le marché des produits structurés de crédit:

En premier lieu, le point de départ de la crise, le marché des crédits hypothécaires et des produits structurés de crédit, n'était pas un marché caractérisé par la transparence.

En effet, les produits structurés de crédit, comme les CDO, les CLO ou encore les CDO squared, constituent un triple problème du point de vue de la transparence, les produits structurés de crédit manquent clairement de 
transparence En effet, ils sontconstitués d'un nombre si important de prêts que cela rend l'identification des risques auxquels le produit est exposé quasi impossible. Ainsi, dans une structure à deux ou trois degrés, voire plus (le CDO squared étant composéentre autre de parts de CDO, eux-mêmes constitués de parts dans des ABS, MBS, ou RMBS, qui sont chacun constitués de plusieurs centaines voireplusieurs milliers de prêts), il est extrêmement difficiled'identifierl'exposition au risque subprime par exempleCelarendla Discrimination des risques impossible, ce qui conduit, en cas de choc,comme cela a été le cas au début de l'été 2007 , à une crise de confiance (du fait de ladispersion de risques inconnus et non mesurables, que les marchés ne saventpas biengérer contrairement au risque mesurable).

Un autre marché a présenté certaines imperfections, qui sont venues aggraver la crise de confiance et donc la contagion financière Il s'agit du marchédes CDS (CreditDefault Swap).Ce marché a faitParticulièrement parler delui au moins à trois reprises, avec la quasi-faillite de BearSterns, la faillite de LehmanBrothers et le sauvetage d'AIG,Mis au point dans les années 1990 par les équipes de JP Morgan.

Ces CDS sont des instruments financiers ni régulés, ni transparents, puisque constitués de contrats bilatéraux privés et non d'opérations conclues sur un marché organisé. Instrument de spéculation facile, le CDS permet de faire un pari sur le risque d'un investissement (crédit, obligation) sans financer l'investissement lui même.Cetinstrument s'avère dangereux quand il a été utilisé pour fabriquer desplacements obligataires synthétiques, basés sur une superposition de dérivés de crédit, autour des crédits hypothécaires américains. Certains de ces placements, sous forme d'obligations notées AAA, se sont retrouvés dans les bilans bancaires. L'absence de transparence des dérivés de crédit a ainsi été une des causes de l'extension de la crisedes subprimes, au delà de l'économie américaine, à une grande partie des banques européennes.Les CDS ont donc bien constitué une source de risque de contagion supplémentaire le mode d'échangede gré à gré, de ces contrats a en effet eu deux conséquences. D'une part l'absence de gestion du risque de contrepartie sur ces marchés, en l'absence de chambre de compensation, a réintroduit le risque qu'une contagion de type dominos apparaisse (la rapidité et l'ampleur de la réaction des pouvoirs publics américains aux problèmes de BearSterns et AIG tient précisément à la conscience qu'ilsavaient de ce problème). D'autre part, l'absence de chambre de compensation rend lemarché des CDS complètement opaque aucune information n'est publiquementdisponible sur le montant des expositions des différents vendeurs de protection(13).

2- $\quad$ Le rôle de l'innovation dans la crise des dettes souveraine : 
La crise financière fut d'abord une crise bancaire car touchant le marché interbancaire Si elle n'a pas provoqué la crise économique, elle l'a cependant amplifiée etau fil des difficultés, après les interventions massives des banques centrales, les états sont intervenus pour relancer les économies. Aujourd'hui, la crise a muté elle s'est transformée d'une crise de l'endettement privé en une crise de l'endettement public qui s'est révélée au départ être celle de la Grèce qui aura impacté les banques mais qui aura également été révélatrice d'autres difficultés dans d'autres paysque nous pouvons schématiser comme suit :

Schéma 1: De la crise des subprimesà la crise des dettes souveraine.

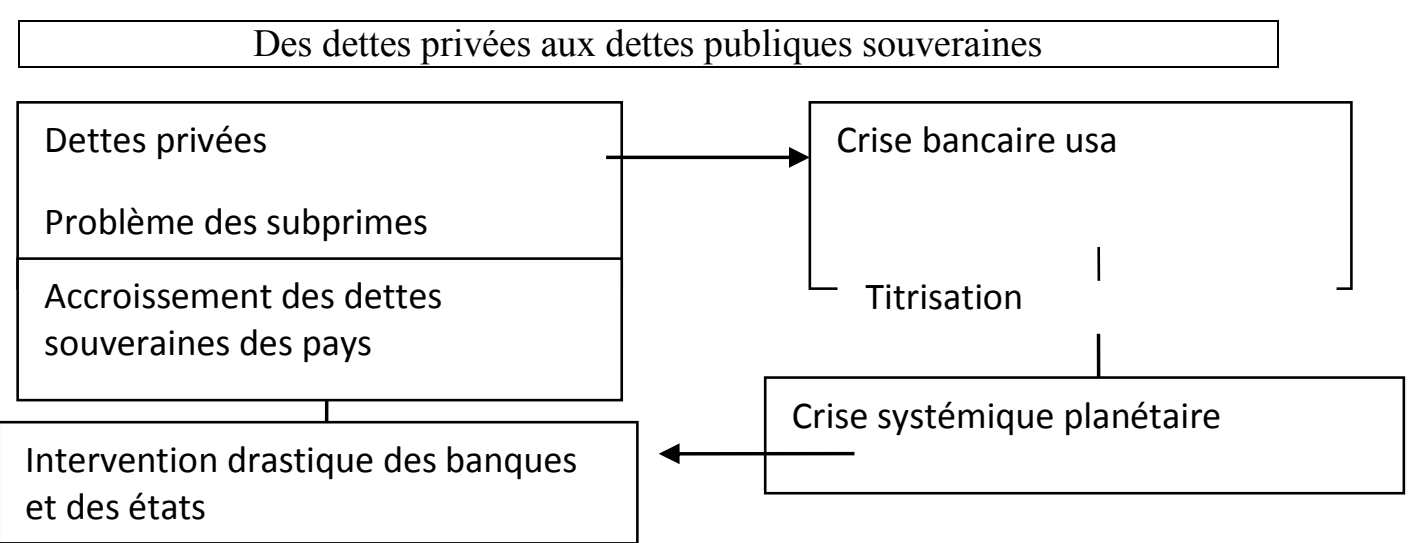

Source : Catherine Karyotis, L'essentiel des banques 2015-2016 2eme éd, édition Gualino, France, 2015 ,P94.

$\mathrm{Au}$ fil des innovations on retrouve aussi les Cds ces produits se sont avérés plus nocifs

encore lors de la crise des dettes souveraines européennespour comprendre ce qui s'est passé en Grèce il faut rappeler qu'un CDS peut être comparé à une assurance souscrite par un créancier, détenteur d'un titre de créance pour se 
couvrir du risque de défaut d'un emprunteur. Le vendeur de la protection qui est supposé assumer le risque en cas de défaut perçoit en échange du service offert une prime à échéance fixe payée par le détenteur du titre qui cherche à se couvrir contre le risque en achetantune protection Cette prime est supposée assurer la contrepartie du remboursement despertes si un événement de crédit fixé dans le contrat se produit.

Le niveau de la prime de risque, qui se négocie sur le marché, est perçu comme une indication de la qualité de crédit de l'actif de référence Plus la prime d'un CDS est élevée, plus l'actif sous-jacent dans notre cas un bon du trésor grec est perçu comme risqué de même, quandla situation d'un emprunteur se détériore, notamment lorsque sa note est dégradée, la prime à verser pour une nouvelle opération de protectionaugmente Les primes fluctuent donc en fonction de l'évolution de la perceptionqu'a le marché de la qualité de crédit de l'actif considéré.

\section{Graphique 1: évolution des Primes de CDS des pays européens}

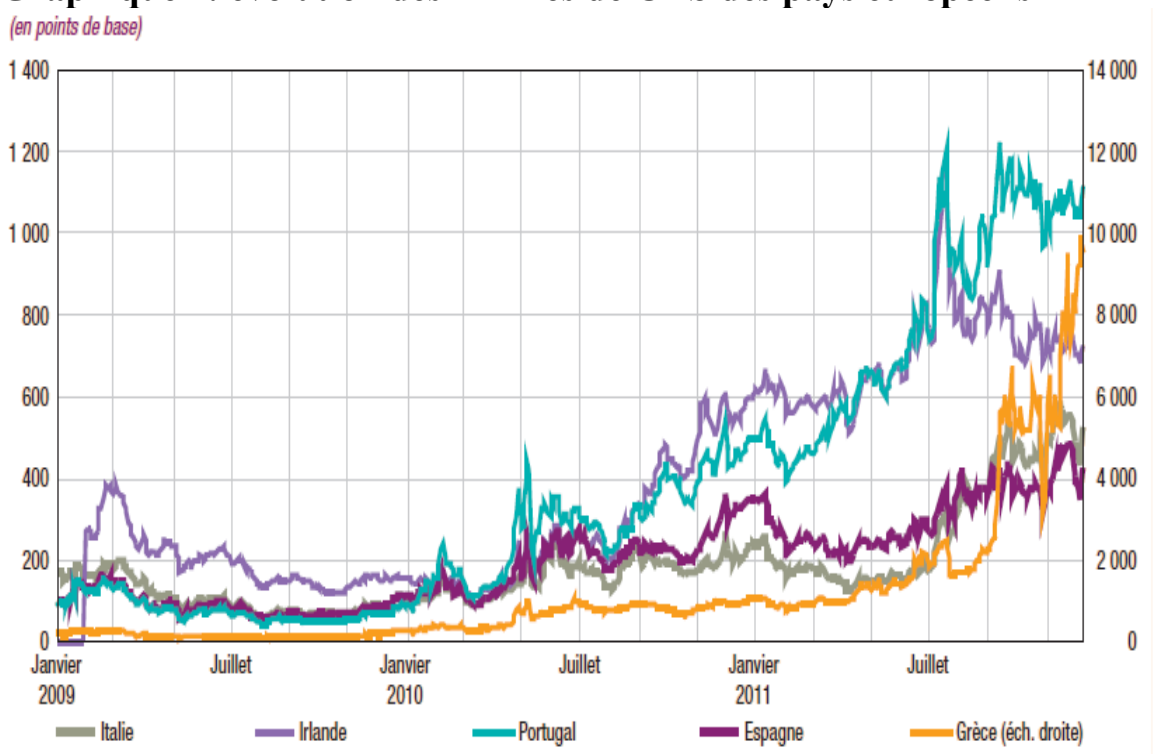

Source : Banque de France, la crise de la dette souveraine, document et débats $N^{\circ} 4$, mai 2012, P32.

Evidemment il existe un marché des CDS non régulé et totalement opaque, sur lesquels les CDS s'échangent dans des contrats de gré à gré.

Dans le cas qui nous occupe, il suffit donc que le risque attaché aux titres de la dette publique grecque augmente pour que la prime de risque revendiquée 
par les vendeurs de protection augmente Leur intérêt est donc que ce risque perçu soit le plus élevé possible de surcroît les CDS sontdes produits autonomes les investisseurs peuvent librement acheter et vendre des CDS nus sur des obligations qu'ils ne détiennent pas de ce fait, les spéculateurs peuvent jouer sur le défaut d'un Etat vis-à-vis duquelils ne sont pas exposés C'est comme si quelqu'un prenait une assurance incendie sur la maison de son voisin ilaurait alors tout intérêt à y mettre le feu pour toucher l'assurance Et c'est bien sûr ce qui advint. La spéculation sur la dette grecque relayée par et appuyée sur les notes toujours plus dégradées des agences de notation a battu son plein les détenteurs de CDS ont ainsi vu lesPrimes qui leur sont allouées monter en flèche, et ce à risque pratiquement nul(14).

-De la réglementation des innovations financière:

La sophistication et la multitude d'instruments financiers CDS et CDO, et autres produits structurés ont quelque peu brouillé les repères, et notamment la capacité de compréhension, d'analyse et donc de contrôle de l'activité de titrisation, où l'information est diluée, tout en favorisant le volume de crédits au détriment de la qualité ce qui présente des anomalies dans la gestion du risque, puisque tant la notation quel'information sur les titres sont floues et parfois contradictoires.

A l'opposé, la titrisation correctement gérée assurerait une dissolution du risque plus efficace tout en proposant des coûts de financement réduits et unchoix de portefeuille plus large La titrisation doit être simplifié, avec la suppression des titres multi étagescomme les CDO pour avoir des titres dont le risque est divisé donc facilement identifiable(15).

La titrisation doit faire l'objet d'un contrôle strict il n'est pas question d'adopter une mesure globale permettant aux banques de titriser sans contrôle quelles dettes sont titrisés et comment sont évaluées et vendues les obligations créées?

Puis de lutter contre l'aléa moral lié à la titrisation les banques devraient supporter le risque des crédits qu'elles accordent, et ne pas uniquement transférer le risque à un tiers via le marché des dérivés avec l'idée d'une obligation de détenir au moins $10 \%$ de la responsabilité sur un crédit accordé(16).

L'organisation des marchés d'actifs serait ainsi segmentée et mieux organisée, ce qui permet une meilleure supervision, que ce soit par les investisseurs euxmêmes ou par un organisme chargé de cette mission de surveillance. Une telle tutelle de marché serait affectée à la surveillance des marchés dérivés et pourrait être doublée d'une chambre de compensation, qui préviendrait les déséquilibres et notamment le risque d'illiquiditéenfin, cela suppose une évaluation performante des actifs c'estàdire consciente des vrais risques par 
les différents acteurs en outre les investisseurs doivent réellement porter le risque lors d'une spéculation, et pas uniquement le transférer à un tiers.

La question de la standardisation des produits de la titrisation est primordiale. Les marchés de certains produits dérivés sont ainsi très peu régulés et les informations sur les titres et les données pertinentes sur un produit dérivés sont très important, à savoir le montant réel du crédit, son échéancier, sa nature risquée ou non ce sont ces informations qui ont manqué lors de la crise des subprimes.

Les CDO ont par exemple vocation à regrouper des crédits toxiques et des crédits sains, afin de diluer le risque c'est ce mélange qui est justementdissimulateur du risque réel attenant à un crédit c'est un des points de départde la crise des subprimes. Il est nécessaire de créer plusieurs marchés d'obligations, dans lesquels chacune des tranches d'un CDO serait négociée : le risque est ainsi connu et donc mieux appréhendé. Les différents Asset-Backed Security ABS liés aux CDO seraient standardisés et leur gestion différenciée selon la qualité les plus risqués seraient centralisés et normalisés, en plus d'être régulés par une chambre de compensation. L'objectif principal est d'éviter le risque systémique et de limiter automatiquement les leviers financiers excessifs et la concentration des positions dans le même sens.

Ou encore la crise de la dette grecque 2010 prouve que La standardisation doit permettre une complète perception des risques et des rendements lorsqu'un investisseur acquiert un produit dérivé.

Pour la question de la régulation du marché des CDS, là encore la standardisation est nécessairells doivent être régis par des normes internationales précises, et échangés surdes marchés spécifiques, différenciés selon le type de CDS. Il serait bon d'envisager la création de chambres de compensation, qui garantiraient la liquidité des contrats, par l'exigence de dépôts préalables aux prises de position et compensés en fin de journée(17).

Parailleurs il serait nécessaire de conditionner l'achat d'un CDS à la détention de l'obligation sous-jacenteen effet, le fait de détenir une assurance sur un bien que l'on ne possède pas pose un problème d'aléa moral, VoireD'intéressement à la réalisation du risque. En outre, il serait opportun d'interdire les transactions Over theCounter (OTC), c'est-à-dire de gré à gré, pour les institutionnaliser et supprimer leur opacité(18).

-Bâle III :

En 2010, le Comité de Bâle publie son troisième accord un ensemble actualisé de règles qui permettraient d'assurer une meilleure solidité et stabilité du secteur bancaire et qui garantiraient une moindre gravité des crises futures dès lors, quel eut dû être le niveau de fonds propres minimum pour absorber les pertes enregistrées en 2008 ? Certaines recherches avancent que la moyenne des pertes des banques durant la crise se situait à $5 \%$ de leurs actifs pondérés, d'autres études indiquent qu'avoir $10 \%$ de fonds propres offrirait un avantage 
significatif en termes de solidité, tandis que, lors de la dernière crise, un taux de $16 \%$ aurait permis d'absorber la plupart des pertes individuelles et un taux de $24 \%$ aurait permis d'absorber presque toutes les pertes dues aux crises bancaires depuis 1988, Au-delà d'une nouvelle définition des fonds propres réglementaires, Bâle III prévoit une meilleure couverture des risques enparticulier de contrepartie l'introduction d'un ratio de levier harmonisé auniveau international, la constitution decoussinscontra-cycliques, l'introduction de deux ratios deliquidité ratios court terme et financement stable, et cherche àréduire la pro-cyclicité par le passage d'un modèle comptable de pertes encourues à une méthode des pertes attendues(19).

D'autres instruments financiers qui étaient jusquelà considérés comme des quasi-fonds propres, ne le seront plus à partir de l'entrée en vigueur de Bâle3 Les leçons des subprimes ayant été retenues, il faudra par exemple davantage tenir compte des produits dérivés et des opérations de titrisation pour apprécier les engagements d'une banque enfin, les ratios minimum à respecter augmenteront eux aussi le ratio de fonds propres durs passera de $2 \%$ à $4,5 \%$ des engagements pondérés, et il faudra lui ajouter un coussinsupplémentaire de conservation de 2,5\% Par ailleurs, Bâle 3 revient sur la sophistication trop grande de Bâle1 et 2 en matière de pondération des risques il ajoute aux ratios pondérés un ratio délibérément simple, le ratiode levier, qui rapportera les fonds propres durs d'une banque au total de ses expositions non pondérées au bilan et au hors-bilan. Ce ratio devra dépasser 3\% enfin, Bâle3 ne s'occupe pas seulement de la solvabilité il introduit des normes de liquidité ilobligelesbanquesà disposer d'un matelas minimal d'argent immédiatement disponible pour pouvoir temporairement faire face à un assèchement du marché interbancaire (situation connue en 2008). Il s'agit de ne pas obliger la Banque centrale à inonder les banques en liquidités à bon marché(20).

III. Quels Impacts des innovations financières sur l'économie algérienne ? -Un secteur financier déconnecté du system financier international :

D'abord épargnée par les effets directs de la crise financière en raison de la faible intégration de sonsystème financier à la finance internationale en ce qui concerne le réseau bancaire, la plupart des banques ont des capitauxMajoritairement nationaux lesquelles contrôlent plus de $90 \%$ du marché bancaire Il est vrai que certaines banques étrangères (Société Générale, BNP Parisbas, Natexis...) implantées ont leur banque mère touchée par la crise,cependant, les répercussions sur le système bancaires algérien ne sont pas significativescompte tenu de leur part de marché, qui demeure faible, et au final de la maîtrise de la situation par les banques mères.

Par ailleurs, la plupart des banques sont dans les textes généralementuniverselles (l'universalité des banques a été introduite en Algérie 
en 1990) mais dans la réalité, il ne s'agit pas de grandes banques ayant une activitéinternationale importante, quand elles interviennent sur les marchés internationaux, leursactivités sont encadrées elles ne font pas recours aux produits dérivés et autres techniques detitrisation à l'origine de la crise tels que les MBS, CDS, ou CDO pour le placement de leursressources en devises elles ne sont donc pasconcernées, à l'instar des gigantesques banquesD'affaires mondiales, par l'acquisitiondes titres toxiques, qui demeurent l'une desgrandes courroies de transmission de la crise au reste du monde. En outre, étant soumises àune réglementation stricte et compte tenu du contexte économique en développement danslequel elles évoluent, les banques algériennes s'adossent plutôt sur une collecte deressources stables pour soutenir l'investissement réel et non l'investissement financier et laspéculation pour la recherche de gains faciles.

N'ayant donc pas investi dans les marchés hypothécaires à haut risque et ne détenantpas de titres représentatifs des crédits subprimes, mais plutôt tournées vers le financementdu secteur réel, les banques algériennes ne connaissent pas de pertes et encore moinsde faillites liées à la crise financière mondiale(21).

En ce qui concerne le marché financier l'Algérie, elle s'est certes dotée, dans le cadre des réformeséconomiques, d'un marché financier depuis décembre 1993 par le décret législatif $n^{\circ} 93 / 10$ du 23/05/1993, mais depuis son démarrage en 1998, celui-cidemeure encore à l'état embryonnaire, la capitalisation boursière demeure marginale et ne dépasse pas actuellement $0,25 \%$ du PIB.

Cette faiblesse est le résultat du nombre de titres de capital cotés, et par leur cours relativement réduit. La bourse d'Alger est aussi caractérisée par un total détachement vis-à-vis des marchés financiers mondiaux, il n'ya point d'investissements en portefeuille étrangers alors que les lois bancaires (loi $90 / 10$ et l'ordonnance $\mathrm{n}^{\circ} 03.11 \mathrm{du} 26 / 08 / 2003$ relatives à la monnaie et crédit) prônentla liberté de mouvements de capitaux(22).

-Impacts financiers directs sur la valeur des actifs de la Banque d'Algérie :

Dans son rapport 2009, la Banque d'Algérie affirme que sa politique de placements des réserves de change a contribué à atténuer l'effet de la forte contraction des recettes d'exportation d'hydrocarbures sur le niveau des réserves officielles de change de

L'Algérie, mais aucun détail n'est donné sur cette politique. Si on tient compte du fait que ce portefeuille se compose d'une majorité d'actifs dits non risqués peu rémunérateurs (dont une bonne part de bons du Trésor américain) et d'une part d'actifs risqués, on peut supposer qu'il y a pu avoir des pertes d'abord sur les actifs risqués et ensuite sur ceux en dollars du fait de la baisse du taux de change de cette devise ce qui a d'ailleurs amené les autorités monétaires à sécuriser ces placements.

En effet, en 2009, les réserves officielles de change atteignirent le niveau record de plus de 147 milliards de dollars. Cependant, en termes de flux, 
conséquence de labaisse des recettes provenant de la vente des hydrocarbures, les montantsengrangés en 2009 dépassaient à peine 4 milliards de dollars, contre prèsde 33 milliards en 2008.

l'accumulation d'importantes réserves de change entre 2004et 2008 et le remboursement par anticipation de la dette extérieureau cours des deux années (2005-2006) précédant la crise financièreinternationale ont permis largement à limiter lerisque de contagion financière pure(23).

-La baisse des IDE face a une résilience:

L'Algérie a enregistré une baisse de $60 \%$ des investissements étrangers en 2009 par rapport à l'année 2008 cette baisse est-elle la conséquence des mesures d'encadrement des investissements directs étrangers prises dans le cadre de la loi de finances complémentaire 2009 ou est-elle liée àla conjoncture mondiale morose ? En tout cas, le FMI s'est posé la question des effets négatifs de ce recul sur l'attractivité de l'économie algérienne. Quand on regarde l'évolution des IDEen Algérie en 2009 et au début 2012 on observe, dans le secteur horshydrocarbures et hors secteur financier, une baisse d'environ de $60 \%$ en 2009par rapport à 2008 .

Tableau n ${ }^{\circ} 1$ Evolution des Flux nets d'IDE vers l'économie algérienne 2007-2012MUSD

\begin{tabular}{|l|l|l|l|l|l|l|}
\hline & 2007 & 2008 & 2009 & 2010 & 2011 & 2012 \\
\hline Algérie & 1662 & 2593 & 2746 & 2264 & 2571 & 1484 \\
\hline
\end{tabular}

\section{Source : Trésor direction générale, Les investissements directs étrangers en Algérie en 2012, novembre 2013, P2.}

en 2010, alors que dans beaucoup de pays émergents, on observe une reprise assez importante des flux de capitaux, on n'a pas observé un tel rebond en Algérie. Selon le Forum économique mondial 2012-2013 dans son rapport annuel relatif au climat d'affaires et d'investissement, tous les indicateurs de la compétitivité économique montrent un recul important enregistré dans l'environnement des affaires et de l'investissement en Algérie. Avec unPNB de 190 milliards de dollars, l'Algérie reste toujours une destination difficile et non préférée par les investisseurs étrangers. Il faut reconnaître que les dernières mesures contenues dans les lois de finances complémentaires de 2009 et 2010 ont laissé des traces sur le volume des IDE entrants. Cela s'est amplifié avec lacrise mondiale depuis 2008, et la contraction mondiale qui s'en est suivie.

-Les retombées sur les échanges extérieurs:

L'essentiel des effets du choc provoqué par la chute du prix des hydrocarbures au quatrième trimestre 2008 s'est reporté sur l'année suivante, avec une baisse de $42,5 \%$ des recettes d'exportation d'hydrocarbures la dégradation brutale du 
solde des échanges extérieurs s'est traduite par une forte contraction des excédents l'excédent commercial chuta de 19,75 à un milliard de dollars, tandis que celui ducompte courant extérieur passa de 34,45 à 0,41 milliard de dollars. Lesimportations de biens et services, qui croissaient depuis quelques annéesà un rythme soutenu à la faveur du programme de relance de l'économie,ont connu une envolée en 2008, aggravée par la flambée des prix decertains biens alimentaires. Cet emballement des importations, dans lecontexte de crise financière internationale, a alarmé les pouvoirs publicsqui ont très vite réagi par des mesures visant à contenir le niveau desimportations de biens et services(24).

-Les répercussions fatales sur le budget de l'État :

La contraction de la demande internationale sur les produits de base et son effet sur le revenu des exportations d'hydrocarbures ont impactéla ressource publique par le canal de la fiscalité pétrolière. En 2009, pourla première fois depuis la création du fonds de régulation des recettesen 2000, le budget est calculé avec un prix de référence du baril (37dollars) supérieur à celui du marché, donc avec un déficit programmé.Ce déficit a pu être épongé par les réserves accumulées dans le fonds de régulation des recettes et le choc a été amorti.

Cependant, les montants engrangés dans le fondsen 2009 ayant chuté à 400675 millions de dinars (contre 2288 159en 2008), les disponibilités avant prélèvements pour 2009 ne représententque 4680747 millions dedinars (contre 5503690 en 2008). Finalement,parce qu'en 2009 il n'y a pas de remboursement (de la dettepublique oudes avances de la Banque centrale) et que le déficit du Trésor est moindrequ'en 2008 (364 282 millions de dinars contre 1223 617), les réservesaccumulées dans le fonds de régulation des recettes vont se stabiliser à 4316465 millions de dinars(contre 4280073 en2008).

-Forte dépendanceaux hydrocarbures risque systémique et syndrome de 'maladie hollandaise' :

Avant la crise, les fortes recettes engrangées par l'Algérie suite au prix élevé du pétrole lui ont permis d'entreprendre de vastes programmes d'investissements publics mais La réduction des exportations des produits énergétiques (pétrole et gaz dont le prix est indexé) qui s'expliquent par l'effet volume mais aussi par l'effet prix. Il s'avère que la demande mondiale de pétrole accuse, selon l'Agence Internationale de 1'Energie, un net recul (-2,9\%) en 2009. Aussi, le pétrole dont le prix du brent était de $140 \$$ à fin juillet 2008 du fait de la forte demande des pays émergents (notamment chinoise), des tensions anticipées sur les marchés pétroliers mais aussi de lalente réaction de l'offre, a chuté à moins de $60 \$$ en septembre 2008 puis à moins de 40 \$au début février 2009, etse situe à moins de $60 \$$ le baril en juillet 2009, il enregistre ainsi un effondrement de plus de $50 \%(25)$. 
Au moment où l'Algérie doit faire face à une nouvelle crise pétrolière, l'hyper dépendance de son économie aux hydrocarbures fait craindre un risque systémique, si les crises de 1998 et 2009 ont été absorbées à la faveur d'une reprise rapide des cours pétroliers, les amortisseurs mis en place (désendettement, épargne budgétaire et accumulation de réserves de change) pourraient s'épuiser rapidement Le contexte actuel est bien plus défavorable, pour au moins trois raisons.

En premier lieu, la dernière crise pétrolière n'est pas de même nature que les précédentes. La baisse brutale des cours de près de $50 \%$ au cours du second semestre 2014 s'explique moins par le ralentissement de la demande mondiale que par une mutation du marché énergétiquemondial (essor des énergies renouvelables et des hydrocarbures non conventionnels) Le consensus des experts s'établit à un cours durablement compris entre 60 et 75 dollars.

À la chute des cours s'ajoutent de plus la stagnation de la production et des exportations pétrolières (de 1,371 à 1,202 milliard de barils produits entre 2007 et 2013), et la baisse sensible des exportations gazières (de 50 à 44 milliards de mètres cubes entre 2010 et2014cette baisse résulte d'une déplétion des réserves (due à une exploitation intensive dans la dernière décennie), d'un recul des investissements d'exploration et d'une explosion de la consommation intérieure, qui devrait passer à 50 milliards de mètres cubes en 2017-2020.

À court terme, les marges sontétroites à moyen et long termes, incertaines.

En troisième lieu, les amortisseurs financiers tendent à s'épuiser rapidement. Le fonds de régulation des recettes,après avoir atteint son plus haut niveau en 2012 (près de65 milliards de dollars), décroît de $1,5 \%$ en 2013 et de près de 20 $\%$ en 2014 si les cours pétroliers restent stables aucours actuel, le fonds de régulation des recettes pourrait être totalement asséché en moins de deux ans de même, les réserves de change, après avoir atteint leur plus haut niveau à 194 milliards de dollars (soit près de 34 mois d'importations) en 2013, se sont contractées à 178 milliards en $2014 \mathrm{Au}$ cours du seul mois de janvier2015, elles auraient diminué de 11,6 milliards de dollars, soit la plus forte baisse mensuelle depuis plus 30 ans. Selon le Fonds monétaire international(FMI), à ce rythme, les avoirs en devises pourraient être épuisés en 15 mois(26).

\section{Conclusion :}

Les objectifs de ce travail étaient multiples tout d'abord, nous avons cherché à établir un ancrage théorique solide à l'aide de la littérature académique pour analyser le phénomène de l'innovation en finance. Il en a résulté une approche extensive de l'innovation financière, qui peut être définie comme un processus de changement dans la nature où le fonctionnement des marchés, des institutions ou des instruments financiers. Pour appréhender les facteurs et les dynamiques qui président aux processus de changement dans les systèmes financiers, nous avons préféré les approches qui proposent les causes de 
l'innovation financière, telles que les théories de ross et de Silber qui font des comportements innovants une riposte des agents privés aux contraintes et aux imperfections du marché, résultant notamment de la réglementation.

L'utilisation du cadre théorique de la dialectique de la réglementation nous a ensuite permis d'analyser le rôle qu'ont joué les interactions entre réglementations et innovations dans le déclenchement de la crise. Les coûts associés à certaines réglementations financières américaines ont donné lieu au développement de stratégies de contournement de la part des institutions financières, qui reposaient largement sur des innovations financières complexes exploitant les failles de la régulation. Des innovations telles que les crédits subprimes, la titrisation ou les dérivés de crédit ont joué un rôle central dans le déclenchement et la propagation de la crise, ces produits se sont avérés plus nocifs encore lors de la crise des dettes souveraines européennes. Ces instruments permettent de spéculer surla mort d'un émetteur, quand bien même cet émetteur n'est autre qu'un pays, la Grèce par exemple. Le rôle de la réglementation prudentielle, des autorités de marché et des autorités monétaires ont été repensés. Les trois séquences de la dialectique de la réglementation (réglementation, contournement, reréglementation) se sont donc illustrées dans la crise financière. Par l'effort de régulation du shadowbanking La création de la chambre de compensation des produits dérivés etl'instauration de contreparties centrales sur les marchés de dérivés, l'introduction de ratios de liquidité a travers l'évolution du rôle de bale 3 ont modifié le fonctionnement des marchés financiers et peuvent à ce titre être qualifiés d'innovations règlementaires.

Enfin, il est à conclure dans cet article que l'économie algérienne fut peu touchée parla crise grâce à ses réserves en devises et sa faible intégration à lafinanceinternationale ce qu'il a sauvé de la contagion Compte tenu de lapetite taille du système financier de l'Algérie, et d'une intégration limitée aux marchés financiers internationaux et aux produits de l'innovation financières CDO CDS titrisation sauf que la trop forte dépendance des ressources extérieures des seuls hydrocarbures et la faible diversification de l'appareil productif industriel exposent l'économie algérienne à un risque de récession et de contraction sévère des échanges en cas de forte baisse de la demande d'hydrocarbures, ce qui pose la question comment redéployer la stratégie de développement à l'échelle de l'économie nationale, dans un contexte d'instabilité de l'économie internationale ? Cette crise est l'occasion d'abandonner les images d'Épinal sur l'ouverture, la libéralisation et le marché pour une vision plus complexe intégrant lanécessité de maintenir et de pérenniser des mécanismes protecteurs contre les chocs externes.

\section{References bibliographiques:}


1. W.Frame, S. White,Empirical Studies of Financial Innovation: Lots of TalkLittle Action?, Federal Reserve Bank of Atlanta, Working Paper, $\mathrm{N}^{\circ} 12$, 2002, P62.

2. J .Van Horne, Financial Innovations and Excesses, Journal of Finance, Vol 40, N³, $1985, \mathrm{P} 620$.

3. W. Silber,The Process of Financial Innovation, American Economic Review, Vol 73, N², 1983 , P 89.

4. J. Abraham, Innovation Financière et Croissance Economique, Revue d'Economie Financière, $\mathrm{N}^{\circ}$ 2, 1988,P 69.

5. Zvi. Bodie, R. Merton, Finance 3eme éd, édition Pearson Education, France, 2001, P41.

6. S. A Ross,Institutional Markets, Financial Market and Financial Innovation, Journal of Finance, Vol44, N³, 1989,P 541.

7. Catherine Karyotis, L'essentieldes banques 2015-2016 2eme éd, édition Gualino, France, 2015 , P93-94.

8. Pascal-Marie Deschamps, Pierre de Lauzun, Philippe Tibi, Les marchés font-ils la loi ? Réponses aux idées reçues et aux solutions toutes faites, édition revue banque, Paris, 2012, P59.

9. Eric Briys, HenriBourguinat, L'arrogance de la finance : Comment la théorie financière a produit le krach, édition La Découverte, Paris, 2009, P29.

10.B. Eichengreen, Dix questions à propos de la crise des prêts subprime, Revue de la stabilité financière, Banque de France, $n^{\circ} 11$, février2008, P18-29.

11.Bertrand Jacquillat, Vivien Levy-Garboua, Les 100 mots de la crise financière, édition Presses Universitaires, France, 2013, P24.

12.PaulJorion, La crise Des subprimes au séisme financier planétaire, édition Fayard, Paris, 2008, P62.

13.R.JCaballero, A. Krishnamurthy, 2008, Les chaises musicales : uncommentaire sur la crise du crédit, Revue de la stabilitéfinancière, Banque de France, $N^{\circ} 11$, février2008, P16-20.

14.BenjaminCoriat, Christopher Lantenois,La Dette Souveraine Grecque Prise $\mathrm{Au}$ Piège De $\mathrm{La}$ zone euro 2011[Disponiblesur:http://atterres.org/sites/default/files/L\%27imbroglio\%20gr ec.pdf. P15consulté le 12/10/2015.

15.MichelleAglietta, SANDRA Rigot, Crise et rénovation de la finance, édition Odile Jacob, Paris, 2009, P141.

16.Paul Washer, Secret Finance : Le grand Krach, édition Genèse, Bruxelles, 2011, P64.

17. Olivier Klein, Quelles réformes pour limiter l'instabilité financière ? Dix propositionsRevue d'Economie Financière, Nº94, juin 2009, P303. 
18. M.Chesney, " Il faut réformer les bombes financières que sont les CDS ", Les Echos, édition en ligne, 30 mars 2009. [Disponible sur: http://www.lesechos.fr/info/analyses/4847722-il-faut-reformer-les-bombesfinancieres-quesont-les-cds.htm] consulté le 05/10/2015

19. Catherine Karyotis,La crise en 40 concepts clés, édition revue banque, Paris, 2013, P95.

20.olivier Lacoste,Les crises financière Histoires mécanismes et enjeux 2eme édition, édition Eyrolles, Paris, 2015, P173.

21.BouzarChabha, Les Conséquences De La Crise Financière Sur LesPays Du Maghreb , colloque International ,Crise financière internationale Ralentissement économique Mondial Et Effets sur les économies euromaghrébine, Université Abderrahmane Mira de Bejaïa, Octobre 2009,P4 22.Abdelatif Kerzabi, Abdelatif Chelil,Mohamed Saidani, Crise Financière Et Son Impact Sur Les économiesMaghrébines, colloque international Crise Financière Internationale, Ralentissement économique mondialetEffets sur les économies euromaghrébine ,Université Abderrahmane Mira de Bejaïa , Octobre 2009 ,P4.

23.Fatiha Talahite, Ahmed Hammadache, L'économie Algérienne D'une Crise À L'autre, revue Maghreb - Machrek, édition ESKA, N 206, 2010,P18.

24.Fatiha Talahite, Ahmed Hammadache, L'économie Algérienne D'une Crise À L'autre, op. cit, P 18-19.

25.MihoubMezouaghi, L'économie Algérienne : Chronique D'une Crise Permanente revue politique étrangère, edition Institut français des relations internationales (IFRI), $\mathrm{N}^{\circ} 3,2015, \mathrm{P} 12-13$.

26.Ali Bentabet, globalisation et gouvernance d'entreprise, des contres exemples en Algerie ; revue des sciences de gestion Paris , no 259, 2014,P13. 